\title{
Impaired haematopoietic stem cell differentiation and enhanced skewing towards myeloid progenitors in aged caspase-2-deficient mice
}

\author{
Swati Dawar ${ }^{1}$, Nur Hezrin Shahrin ${ }^{1}$, Nikolina Sladojevic ${ }^{1}$, Richard J D'Andrea ${ }^{1}$, Loretta Dorstyn ${ }^{1,3}$, Devendra K Hiwase ${ }^{1,2,3}$ and \\ Sharad Kumar, ${ }^{* 1,3}$
}

The apoptotic cysteine protease caspase-2 has been shown to suppress tumourigenesis in mice and its reduced expression correlates with poor prognosis in some human malignancies. Caspase-2-deficient mice develop normally but show ageing-related traits and, when challenged by oncogenic stimuli or certain stress, show enhanced tumour development, often accompanied by extensive aneuploidy. As stem cells are susceptible to acquiring age-related functional defects because of their self-renewal and proliferative capacity, we examined whether loss of caspase-2 promotes such defects with age. Using young and aged Casp2 ${ }^{-/}$ mice, we demonstrate that deficiency of caspase-2 results in enhanced aneuploidy and DNA damage in bone marrow (BM) cells with ageing. Furthermore, we demonstrate for the first time that caspase-2 loss results in significant increase in immunophenotypically defined short-term haematopoietic stem cells (HSCs) and multipotent progenitors fractions in BM with a skewed differentiation towards myeloid progenitors with ageing. Caspase-2 deficiency leads to enhanced granulocyte macrophage and erythroid progenitors in aged mice. Colony-forming assays and long-term culture-initiating assay further recapitulated these results. Our results provide the first evidence of caspase-2 in regulating HSC and progenitor differentiation, as well as aneuploidy, in vivo.

Cell Death and Disease (2016) 7, e2509; doi:10.1038/cddis.2016.406; published online 1 December 2016

Caspase-2, a CARD-containing caspase, is the most evolutionarily conserved member of the caspase family. ${ }^{1-3}$ In addition to its function in cell death, it has been shown to act as a tumour suppressor (reviewed in Puccini et al. ${ }^{4}$ ). For instance, CASP2 on human $\mathrm{Ch} 7 \mathrm{q}$ is frequently deleted in haematological malignancies ${ }^{5}$ and reduced CASP2 expression is noted in Burkitt's lymphoma, mantle cell lymphoma, chronic lymphocytic leukaemia (CLL) and hairy cell leukaemia, and correlates with poor prognosis in acute myeloid leukaemia (AML) and acute lymphocytic leukemia (ALL). ${ }^{6-8}$ In The Cancer Genome Atlas (TCGA) (http://cancergenome.nih. gov/) and BloodSpot databases, lower CASP2 expression is clearly linked to poor patient survival in AML (http://servers.binf. ku.dk/bloodspot/?gene =CASP2\&dataset $=$ normal_human_v2_ with_AMLs).

The correlative observations indicating a role for caspase-2 in human malignancies are supported by experimental evidence in mouse models of tumours. For example, caspase-2 deficiency enhances lymphomagenesis in $E \mu-M y c$ transgenic mice that develop B-cell lymphoma ${ }^{9,10}$ and in ataxia telangiectasia mutated (Atm)-deficient mice that spontaneusly develop thymic lymphoma. ${ }^{11}$ Furthermore, MMTV/c-neu-driven mammary carcinoma, ${ }^{12} \mathrm{~K}$-Ras-driven lung carcinoma ${ }^{13}$ and diethylnitrosamine-mediated hepatocellular carcinoma ${ }^{14}$ also show more rapid develoment of tumours in caspase-2-deficient $\left(\right.$ Casp $\left.^{-1}\right)$ mice. In addition, in vitro studies demonstrate that mouse embryonic flibroblasts (MEFs) derived from Casp2 ${ }^{-1}$ mice become immortalized more readily and show enhanced sensitivity to transformation by oncogenes, including Ras and cMyc. ${ }^{9,15}$ Interestingly though, not all types of tumours are affected by the loss of caspase-2, ${ }^{10,16}$ suggesting that the nature of cell types involved (such as their proliferative capacity) may determine the participation of caspase-2 as a tumour suppressor. One commonly observed feature of caspase-2-deficient tumours and MEFs is enhanced aneuploidy, $4,11,12,14,15,17$ likely because of reduced or inefficient apoptotic removal of aberrant cells in the absence of caspase-2.

In addition to enhanced sensitivity to tumourigenesis, Casp $2^{-1-}$ mice show signs of premature ageing including increased oxidative stress and DNA damage. ${ }^{18,19}$ Our previous work suggests that the ageing phenotype is partly because of increased oxidative stress-induced damage and impaired antioxidant response. ${ }^{18,20}$ In oxidative challenge experiments we observed increased tissue damage, accompanied by higher serum IL- 6 and IL- $1 \beta$ levels in Casp2 $^{-/}$mice compared with wild-type (WT) animals. ${ }^{14,20}$ More recent studies suggest a role for caspase-2 in the regulation of agerelated proteostasis, energy metabolism, lipid metabolism and sex-specific alterations in glucose homeostasis. ${ }^{21,22}$ As loss of caspase-2 increases genomic instability and predisposes mice to various types of tumours we hypothesize that

\footnotetext{
${ }^{1}$ Centre for Cancer Biology, University of South Australia, Adelaide, South Australia 5001, Australia and ${ }^{2}$ Department of Haematology, SA Pathology, Adelaide, South Australia 5000, Australia

*Corresponding author: S Kumar, Centre for Cancer Biology, University of South Australia, Frome Road, Adelaide, South Australia 5001, Australia. Tel: +61 882223738 ; Fax: +61 88222 3162; E-mail: sharad.kumar@unisa.edu.au

${ }^{3}$ Co-senior authors.

Received 14.9.16; revised 26.10.16; accepted 07.11.16; Edited by M Piacentini
} 

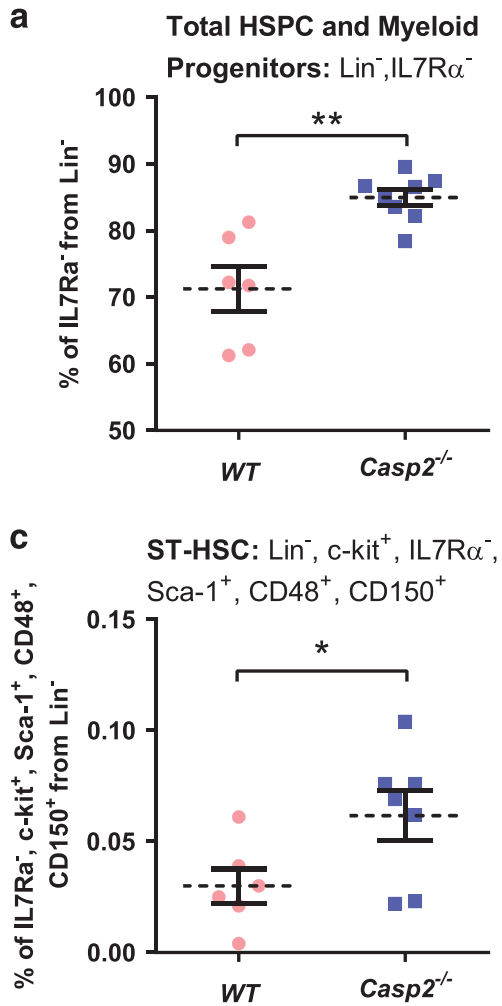

e

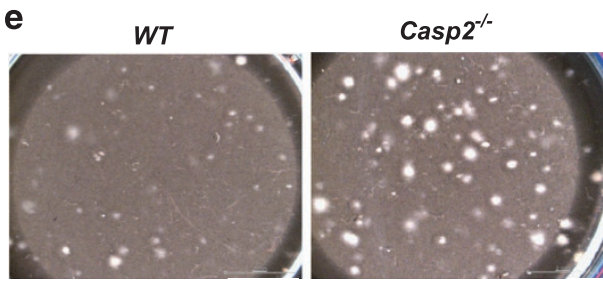

b

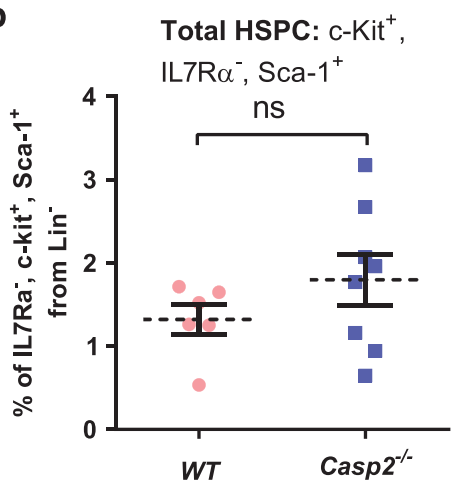

d

d MPP: Lin $^{-}, \mathrm{C}^{-\mathrm{kit}}{ }^{+}, \mathrm{IL} 7 \mathrm{R} \alpha^{-}$,

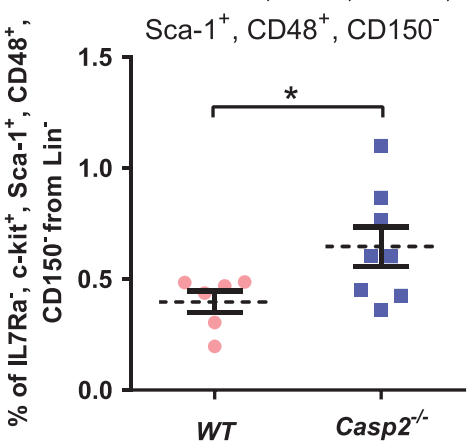

f

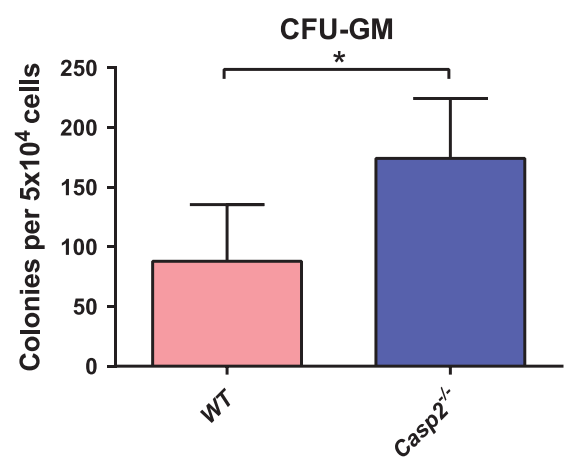

Figure 1 Functional characterization of HSPCs in Casp2 ${ }^{-/}$mice. (a) HSPC and myeloid progenitor cells from Lin ${ }^{-}$, IL7R $\alpha^{-}$bone marrow cells quantitated by flow cytometry. Results were obtained from WT $(n=6)$ and Casp2 $^{-/-}(n=8)$ mice at 24 months. Data represented as mean \pm S.E.M., ${ }^{* \star} P<0.01$. (b) The percentage of total HSPC cells from c-Kit ${ }^{+}$, IL7R $\alpha^{-}$, Sca- $1^{+}$population in the bone marrow from 24-month-old mice followed by (c) ST-HSC and (d) MPP. Results were obtained from WT ( $n=6$ ) and Casp2 $^{-/}(n=8)$ mice at 24 months. Data represented as mean \pm S.E.M. $P$-values are indicated as either non-significant (ns) or ${ }^{*} P<0.05$. (e) LTC-IC assay. Representative image of colonies obtained from 5 weeks of culture of BM cells from WT and Casp2 ${ }^{-1-}$ mice. Scale bar $=50 \mu \mathrm{M}$. (f) Quantitation of colonies obtained from LTC-IC assay for WT $(n=4)$ and Casp2 $^{-/}(n=4)$ mice at 24 months. Data represented as mean \pm S.E.M. ${ }^{*} P<0.05$

caspase- 2 deficiency results in a compromised state that is more susceptible to various oxidative and oncogenic insults, including that associated with ageing.

Stem cells are highly susceptible to acquiring age-related phenotypic and functional changes, such as aneuploidy, DNA damage and skewed differentiation potential, owing to their high self-renewal and regenerative capacities. ${ }^{23,24}$ Therefore, this study examined whether caspase-2 deficiency could exacerbate these age-related changes in the haematopoietic stem cell (HSC) and haematopoietic stem and progenitor cell (HSPC) lineages in the bone marrow (BM). Our data suggest that aged Casp2 $^{-/-}$mice indeed show significant impairment of HSC differentiation and enhanced skewing towards myeloid progenitors compared with WT mice. Furthermore, our data also suggest that caspase-2 deficiency alone is sufficient for increased number of aneuploid cells in the BM of aged animals.

\section{Results}

The CASP2 data in BloodSpot suggest high-level expression in the myeloid lineage including common myeloid precursor cells, granulocyte monocyte progenitors, early and late promyelocytes, myelocytes and megakaryocyte erythoid 
a

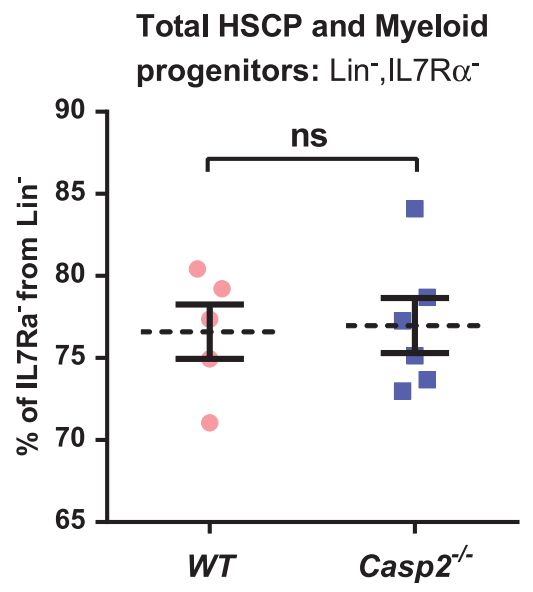

C

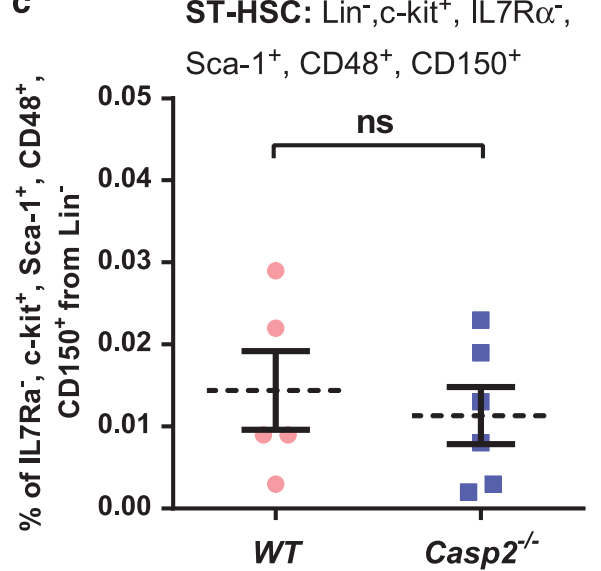

b

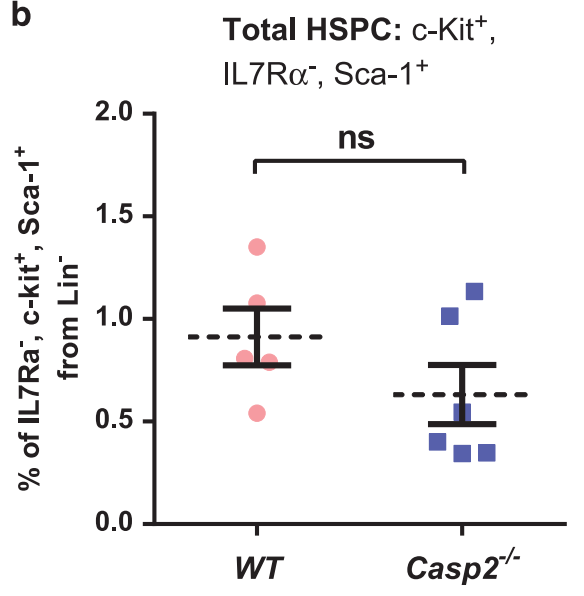

d

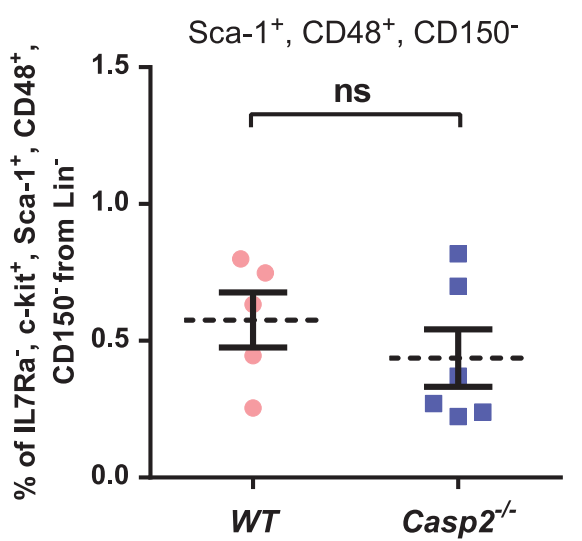

Figure 2 Functional characterization of HSPC in young Casp2 ${ }^{-/-}$mice. (a) The percentage of total HSPC and myeloid progenitor cells (Lin ${ }^{-}$, IL7R $\alpha^{-}$) from BM using flow cytometry. Results were obtained from young WT $(n=5)$ and young $\operatorname{Casp2}^{-/-}(n=6)$ mice. Data represented as mean \pm S.E.M. P-values were non-significant (ns). (b) The percentage of total HSPCs from c-Kit ${ }^{+}$, Lin ${ }^{-}$population in the BM of young mice followed by (c) ST-HSC and (d) MPP. Results were obtained from young WT ( $n=5$ ) and young Casp2 $^{-1-}(n=6)$ mice. Data represented as mean \pm S.E.M. $P$-values are ns

progenitor cells. This observation, combined with a putative role for caspase-2 in haematopoietic tumours, suggested that caspase-2 function may be of particular significance in the haematopoietic lineage.

To test the impact of caspase-2 loss in haematopoiesis with ageing, primary BM cells were isolated from young (4-6 weeks) and aged (18-24 months) Casp2 ${ }^{-1-}$ mice ${ }^{25}$ and assessed for HSPC fractions by flow cytometry ${ }^{26,27}$ and for long-term culture-initiating cell (LTC-IC), BM-derived colonyforming unit granulocyte-macrophage (CFU-GM) and serial replating assays. Though total bone marrow morphology was not substantially different between aged WT and Casp2 $2^{-/}$ mice (Supplementary Figure S1), BM cells from aged Casp $^{-/}$mice had significantly higher HSPC and myeloid progenitors (defined by $\mathrm{Lin}^{-}$, ILR7 $a^{-}$) compared with aged WT mice (Figure 1a and b). Similarly, short-term HSC (ST-HSC) (defined by Lin ${ }^{-}, \mathrm{C}^{-\mathrm{Kit}^{+}}$, ILR7a ${ }^{-}, \mathrm{Sca}-1^{+}, \mathrm{CD} 48^{+}$, and CD150 ${ }^{+}$) and mutipotent progenitor (MPP) (defined by $\mathrm{Lin}^{-}, \mathrm{C}^{-\mathrm{Kit}^{+}}$, ILR7 $a^{-}$, Sca- $1^{+}, \mathrm{CD}_{48}{ }^{+}, \mathrm{CD} 150^{-}$) fractions were significantly higher in aged Casp2 ${ }^{-1-}$ BM cells compared with the aged WT BM cells (Figure 1c and d). In contrast, there were no differences in these BM cell fractions in young WT com- pared with young Casp2 $2^{-/}$mice (Figure $2 a-d$ ). These results were further substantiated by LTC-IC and serial replating assays to assess HSPC self-renewal and differentiation capacity.

Assay of total BM from aged Casp- $2^{-/}$mice was consistent with increased LTC-IC compared with aged WT BM cells (Figure 1e and f). Similarly, serial replating assays of BM mononuclear cells demonstrated a significant increase in burst-forming unit erythroid cells (BFU-E) at first plating for aged Casp $^{-/}$compared with aged WT BM cells. In contrast, CFU-GM and CFU granulocyte, erythrocyte, macrophage and megakaryocyte (CFU-GEMM) frequency did not differ at first plating, but these myeloid progenitors displayed increased replating frequency upon secondary and tertiary plating (Figure 3d-f). Colony numbers from primary, secondary or tertiary plating of BM cells from young WT compared with young Casp2 $2^{-1}$ cells were not different (Figure $4 \mathrm{c}-\mathrm{e}$ ). These results are consistent with caspase-2 deficiency resulting in expansion and enhanced self-renewal of the primitive HSC and myeloid progenitor compartment with ageing. 
a

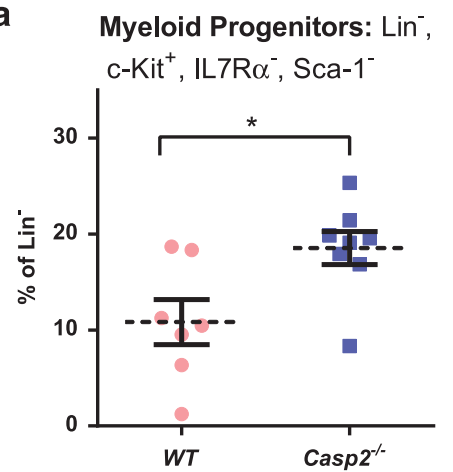

C

CFU-E: Lin ${ }^{-}, \mathrm{c}^{-\mathrm{Kit}^{+}}{ }^{+}, \mathrm{Sca}-{ }^{-}$, $\mathrm{CD}^{-} 1^{-}, \mathrm{FcgR}^{-}, \mathrm{CD} 105^{+}, \mathrm{CD} 150$

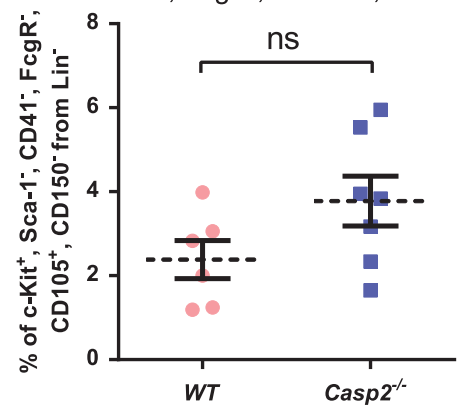

e

CFU- BFU-E

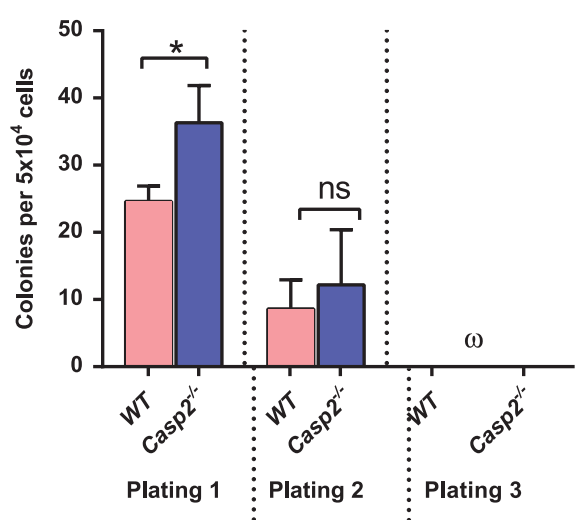

b

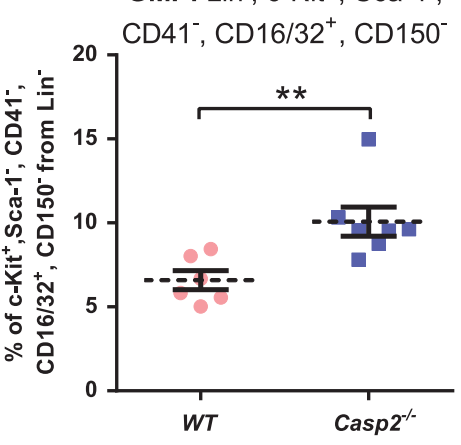

d

CFU-GM

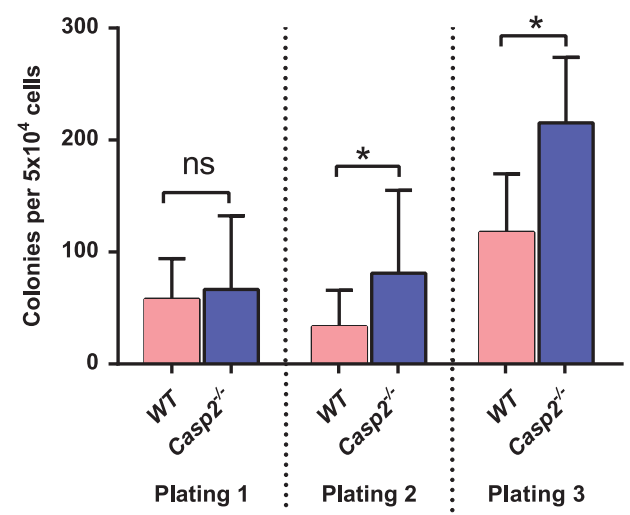

f

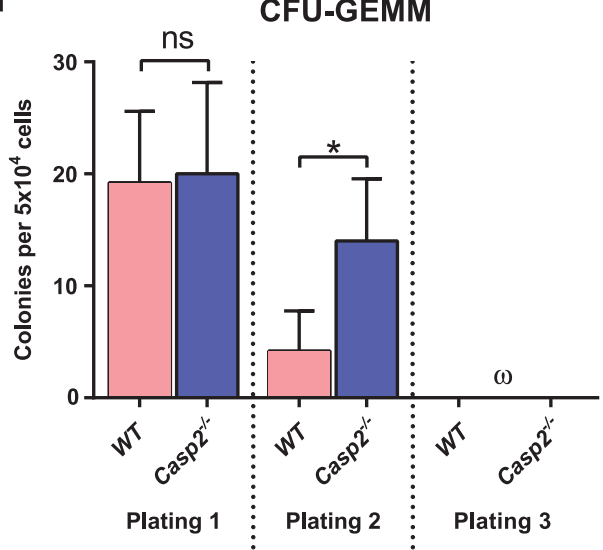

Figure 3 Characterization of myeloid progenitor cells in Casp2 ${ }^{-1-}$ mice. (a) The myeloid progenitor cells from ckit ${ }^{+}, \mathrm{sca1}^{-}$, Lin ${ }^{-}$, IL7R $\alpha^{-}$from bone marrow quantitated by flow cytometry. Results were obtained from WT $(n=7)$ and Casp2 $^{-1-}(n=8)$ mice at 24 months. Data represented as mean \pm S.E.M. $P$-values are indicated with ${ }^{*} P<0.05$. (b) The percentage of GMP and (c) CFU-E in the bone marrow using flow cytometry. Results were obtained from WT $(n=6)$ and Casp2 $2^{-1}(n=7)$ mice at 24 months. Data represented as mean \pm S.E.M. $P$-values are indicated as either non-significant (ns) or ${ }^{* *} P<0.01$. (d) Quantitation of myelo-erythroid progenitor self-renewal from aged bone marrow cells (24 months) using serial colony-forming assay. Colonies scored for CFU-GM obtained from WT $(n=5)$ and $\operatorname{Casp2}^{-1}(n=6)$ mice. Data represented as mean \pm S.E.M. $P$-values are indicated as either ns or ${ }^{\star} P<0.05$. (e and $\mathbf{f}$ ) Colonies scored for CFU-BFU-E (e) and CFU-GEMM (f) obtained from WT ( $n=5$ ) and $\operatorname{Casp2}^{-/-}(n=6)$ mice. Data represented as mean \pm S.E.M. $P$-values are indicated as either ns or ${ }^{*} P<0.05$. The symbol ' $\omega$ ' denotes that no colonies were formed

HSCs are highly susceptible to acquiring precancerous damage such as aneuploidy, DNA damage and skewed differentiation because of their self-renewal and differentiation capacity. ${ }^{24}$ The acquisition of such damage increases with ageing in murine and human bone marrow, ${ }^{28}$ most probably because of their enhanced self-renewal capacity, even though the regenerative capacity of individual stem cells is reduced under stress (i.e., serial transplantation) compared with young HSCs. ${ }^{29,30}$ Normally, the danger caused by mutagenic accumulation in ageing HSC is prevented by the activity of tumour suppressor proteins that sense potentially malignant clones and trigger apoptosis or growth arrest. ${ }^{29}$ It is therefore 
a

GMP: Lin ${ }^{-}, \mathrm{c}-\mathrm{Kit}^{+}, \mathrm{Sca}-1^{-}, \mathrm{CD} 41^{-}$, CD16/32 ${ }^{+}, \mathrm{CD}^{-} 150^{-}$

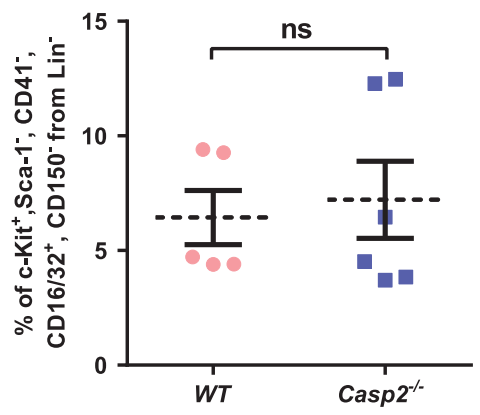

C

CFU-GM

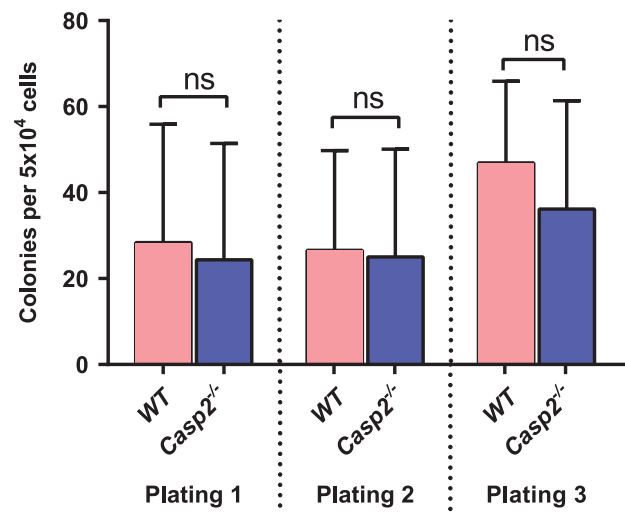

e

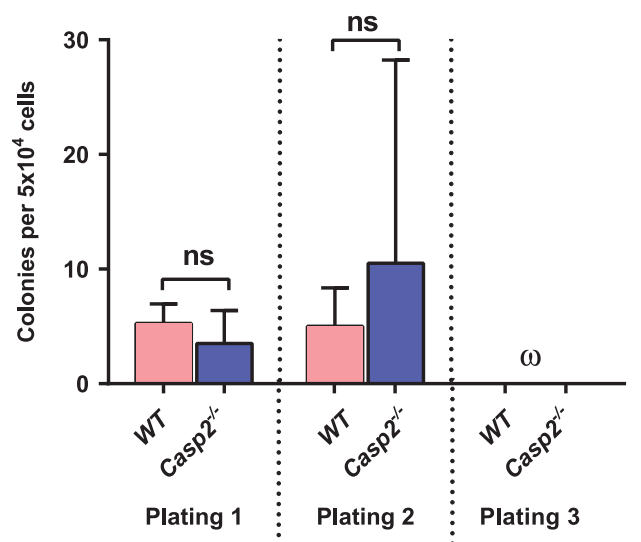

b CFU-E: $\mathrm{Lin}^{-}, \mathrm{C}^{-\mathrm{Kit}^{+}}, \mathrm{Sca}-1^{-}$, $\mathrm{CD}^{-} 1^{-}, \mathrm{FcgR}^{-}, \mathrm{CD} 105^{+}, \mathrm{CD} 150^{-}$

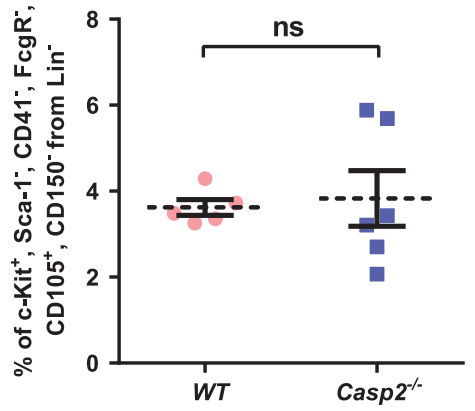

d

CFU-BFU-E

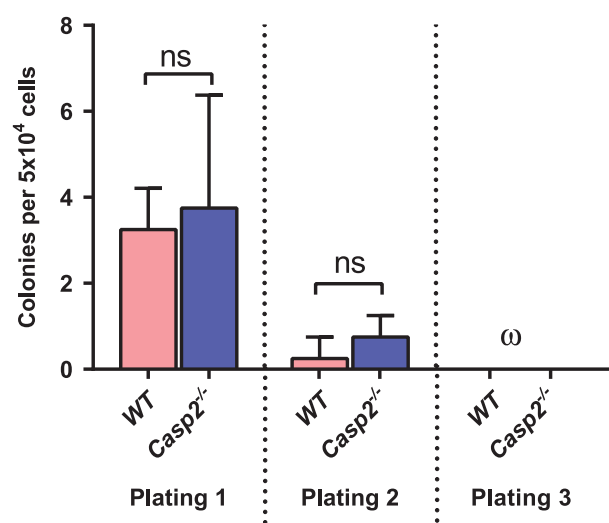

Figure 4 Characterization of myeloid progenitor cells in young Casp2 $2^{--}$mice. The percentage of (a) GMP and (b) CFU-E in the BM quantitated by flow cytometry. Results were obtained from WT $(n=5)$ and $\operatorname{Casp2}^{--}(n=6)$ mice. Data represented as mean \pm S.E.M. P-values are indicated as non-significant (ns). (c-e) Quantitation of myeloerythroid progenitor self-renewal from BM using serial colony-forming assay. Colonies scored for CFU-GM (c), CFU-BFU-E (d) and CFU-GEMM (e) obtained from WT ( $n=5$ ) and $\operatorname{Casp2}^{--}(n=6)$ mice. Data represented as mean \pm S.E.M. $P$-values are indicated as $n s$. The symbol ' $\omega$ ' denotes that no colonies were formed

plausible that caspase-2 may have an important cell death function in HSCs to prevent survival of potential mutagenized cells to prevent onset of haematopoietic malignancies.

Though aged $\mathrm{Casp}^{-1}$ mice show apparently normal total blood counts, and do not develop spontaneous age-related tumours, ${ }^{18}$ caspase-2-deficiency strongly enhances tumour development in various mouse models. ${ }^{9-14}$ This indicates that loss of caspase-2 on its own may not be sufficient to induce tumourigenesis, but increases tumour susceptibility in response to cellular stress (i.e. replicative or oncogenic stress). A recent clinical study established that clonal haematopoiesis, with somatic mutations in genes involved in myeloid malignancies, is increasingly common as people age. ${ }^{31}$ Importantly, these individuals harbouring somatic mutations in myeloid genes had normal blood counts at the time of mutation testing, but had increased risk of 
a

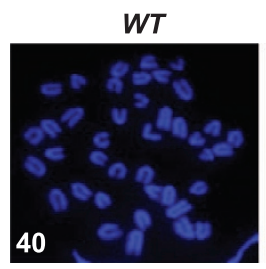

Casp2--

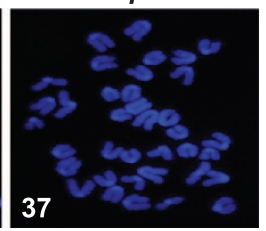

b

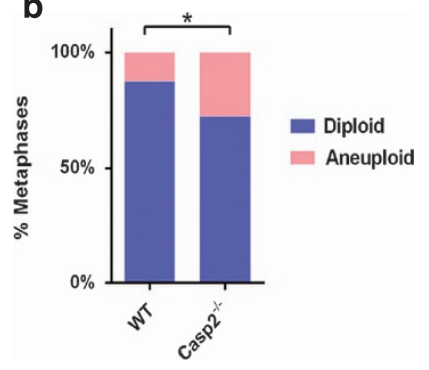

c

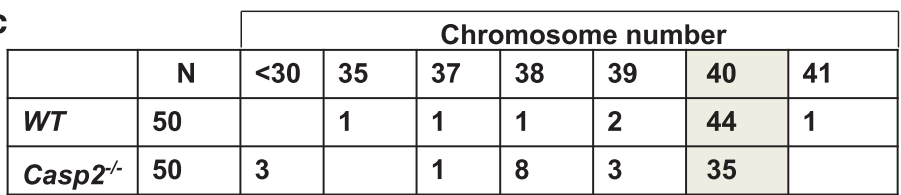

d

DAPI

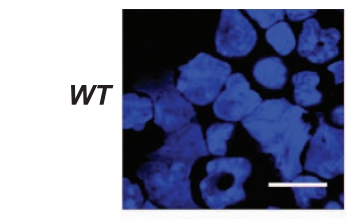

Casp2
YH2AX
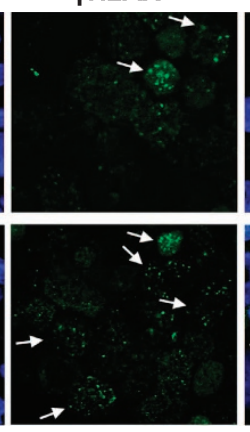

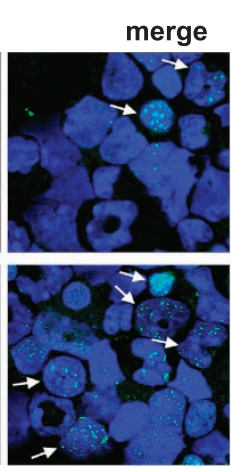

e

Figure 5 Caspase-2 deficiency enhances aneuploidy and DNA damage in primary BM cells with ageing. (a) Representative fluorescent images (magnification of $100 x$ ) of DAPI-stained metaphase spreads, with chromosome counts indicated, from aged WT $(n=4)$ and aged Casp2 ${ }^{-1}(n=4)$ BM cells. (b) Quantitation of metaphases showing frequencies of diploid and aneuploid karyotypes in BM cells from aged WT $(n=4)$ and Casp2 ${ }^{-/}(n=4)$ mice. A total of 50 metaphase spreads were counted per mouse. Data represented as mean \pm S.E.M. and ${ }^{*} P<0.05, \chi^{2}$ test. (c) Table indicating actual chromosome numbers from aged WT and aged Casp2 ${ }^{-/}$BM cells showing reduced cells with diploid number of chromosomes indicated (shaded) and increased aneuploidy in Casp2 ${ }^{-1}$ BM cells. (d) Representative fluorescent images (magnification of $63 \times$ ) of phosphohistone H2AX (Ser139)-stained BM cells from aged WT $(n=4)$ and aged Casp2 ${ }^{-1-}(n=4)$ mice. Arrows highlight cells with multiple $\gamma \mathrm{H} 2 \mathrm{AX}$ foci. Scale bar $=10 \mu \mathrm{M}$. (e) Quantitation of phospho-histone H2AX-positive nuclei of BM cells from aged WT $(n=4)$ and Casp2 $^{-/-}(n=4)$ mice. Data represented as mean \pm S.E.M. and ${ }^{\star} P<0.05$

haematological malignancies during follow-up. Most haematological malignancies arise only after the acquisition of multiple mutagenic events, and the incidence of these haematological malignancies increase with ageing. Gene expression data indicate that CASP2 expression is also lower in AML stem cells compared with normal HSCs, and within an AML cohort, low CASP2 expression is associated with poor outcome of AML cases ( $\mathrm{NCl}$-Cancer Genome Atlas and BloodSpot databases). In addition, CASP2 expression is higher in normal human HSCPs compared with mature monocytes and granulocytes (BloodSpot database), probably essential to eliminate damaged HSCPs.

As the ageing haematopoietic system is associated with expansion of myeloid progenitors, ${ }^{29}$ we assessed the impact of caspase-2 deficiency on these progenitors in aged mice. Analysis of the BM myeloid lineage, including myeloid progenitors (MPs) (defined by $\mathrm{Lin}^{-}, \mathrm{c}-\mathrm{Kit}^{+}, \mathrm{ILR} 7 a^{-}$, and Sca-1-1 ${ }^{-}$, granulocyte macrophage progenitors (GMPs) (defined by $\mathrm{Lin}^{-}, \mathrm{C}^{-K i t^{+}}$, Sca- $1^{-}, \mathrm{CD} 41^{-}, \mathrm{CD} 16 / 32^{+}$, and CD $150^{-}$), megakaryocyte erythrocyte progenitors (MEPs) (defined by c-Kit ${ }^{+}$, $\mathrm{Lin}^{-}$, Sca-1 ${ }^{-}, \mathrm{CD} 41^{-}, \mathrm{CD} 16 / 32^{-}, \mathrm{CD} 105^{-}$, and $\left.\mathrm{CD} 150^{+}\right)$, megakaryocyte progenitors (MkPs) (defined by c-Kit ${ }^{+}, \mathrm{Lin}^{-}$,
Sca- $1^{-}, \mathrm{CD}_{4} 1^{+}$, and $\left.\mathrm{CD} 150^{+}\right)$and erythroid progenitors (CFU-E) (defined by Lin ${ }^{-}$, c-Kit ${ }^{+}$, Sca-1- ${ }^{-}, \mathrm{CD} 41^{-}, \mathrm{CD} 16 / 32^{-}$, $\mathrm{CD}_{105^{+}}$, and $\mathrm{CD} 150^{-}$), was performed in both young and aged mice. Aged Casp2 ${ }^{-1}$ BM cells exhibited significantly increased frequency of myeloid progenitors with a trend towards enhanced GMP populations (Figure $3 a$ and b) compared with aged WT BM cells. Aged Casp2 $2^{-1}$ BM cells also showed a trend of higher erythroid progenitor populations (Figure 3c) compared with aged WT BM cells. Intriguingly, no differences were seen in these progenitor cell populations from young WT and Casp2 ${ }^{-1}$ mice (Figure $4 \mathrm{a}$ and b). This result, combined with the clonal assays in Figure $3 \mathrm{~d}-\mathrm{f}$, suggest that loss of caspase-2 results in a skewing of haematopoiesis towards the myeloid lineage with ageing. These results are consistent with a study demonstrating age-related skewing towards myelopoiesis in adult human BM and further indicate that loss of caspase-2 enhances this phenotype. ${ }^{30}$

As stem cells are susceptible to acquiring mitotic defects with age because of their self-renewal capacity, ${ }^{23,24}$ we examined whether loss of caspase-2 exacerbates agerelated aneuploidy in BM. Indeed, aged Casp2 $2^{-1}$ mice, but not young mice, exhibited significantly enhanced aneuploidy 

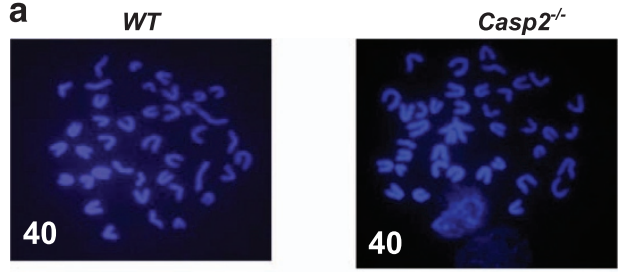

b

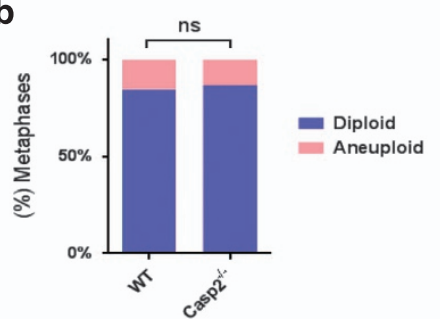

C

\begin{tabular}{|l|l|l|l|l|l|l|l|l|}
\cline { 3 - 9 } \multicolumn{1}{c|}{} & \multicolumn{7}{|c|}{ Chromosome number } \\
\hline WT & $\mathrm{N}$ & 32 & 34 & 35 & 37 & 38 & 39 & 40 \\
\hline Casp2 $^{-/}$ & 50 & & 2 & 1 & & & 5 & 42 \\
\hline
\end{tabular}

Figure 6 Caspase-2 deficiency does not enhance aneuploidy in primary BM cells from young mice. (a) Representative fluorescent images (magnification of $100 \mathrm{x}$ ) of DAPI-stained metaphase spreads, with chromosome counts indicated, from young WT $(n=4)$ and young Casp2 ${ }^{--}(n=4)$ BM cells. (b) Quantitation of metaphases showing frequencies of diploid and aneuploid karyotypes in BM cells from young WT $(n=4)$ and young Casp2 ${ }^{--}(n=4)$ mice. A total of 50 metaphase spreads were counted per mouse. $P$-value is indicated as non-significant (ns). (c) Table indicating actual chromosome numbers from young WT and young Casp2 ${ }^{-1}$ BM cells showing no difference in diploid number of chromosomes (shaded)

in BM compared with WT mice (Figures $5 \mathrm{a}-\mathrm{C}$ and $6 \mathrm{a}-\mathrm{C}$ ). These results indicate that Casp2 ${ }^{-1}$ BM cells are more susceptible to aneuploidy in vivo with ageing and also may explain the association of reduced caspase-2 expression with poor survival in $\mathrm{AML}$, as aneuploidy is a negative prognostic indicator. ${ }^{32,33}$ As ageing is associated with accumulation of DNA damage in $\mathrm{HSC}^{24,34}$ we assessed this and observed significantly enhanced basal $\gamma \mathrm{H} 2 \mathrm{AX}$ foci together with an increased number of $\gamma \mathrm{H} 2 \mathrm{AX}$-positive cells in aged Casp2 ${ }^{-/-}$ BM compared with aged WT cells (Figure $5 d$ and e). Together, these results suggest that caspase-2 deficiency enhances aneuploidy associated with increased DNA damage in BM with ageing.

\section{Discussion}

Caspase-2 deficiency has previously been linked to enhanced haematopoietic malignancy in both humans and mouse models. In this work we now demonstrate a previously unrecognized role for caspase-2 in maintaining HSC homeostasis with ageing that may play a role in premature ageing and enhanced susceptibility to tumourigenesis associated with Casp2 $^{-1-}$ animals. We also show for the first time that caspase-2 loss alone is sufficient for accumulation of aneuploid cells in the BM with age. Our previous work has shown that caspase-2-deficient tumours, as well as MEFs, have increased tendency to become aneuploid and has suggested that this could be because of reduced cell death in a caspase-2-deficient background. ${ }^{11,15}$ Our recent work with live cell imaging of mouse splenocytes ex vivo has demonstrated that caspase-2 is required for deleting mitotically aberrant cells, including aneuploid cells, and that the absence of caspase-2 can promote the long-term survival of such aberrant cells. ${ }^{34}$ Thus, the accumulation of aneuploid cells in the BM of Casp2 $2^{-1}$ animals with age is consistent with the idea that caspase-2-dependent cell death limits aneuploidy, given BM is the compartment where there is continuous cell division, thus increasing the likelihood of these cells having mitotic aberrations.

In the BM, one of the consequences of ageing is that blood cell composition becomes skewed toward myeloid cells, with increased incidence of myelogenous cancer, whereas the main forms of acute leukemia in children is of the lymphoid lineages. ${ }^{28-31}$ Our previous studies have demonstrated that aged Casp $^{-/}$mice exhibit increased reactive oxygen species (ROS) and reduced stress tolerance that is associated with increased DNA damage. ${ }^{15,18,20}$ ROS-related DNA damage is a well-established factor known to compromise HSC renewal and differentiation capacity with ageing. ${ }^{35} \mathrm{~A}$ robust oxidative stress response, together with accurate repair of DNA damage, are critical for HSC maintenance and protection against functional decline of HSCs during ageing. ${ }^{29,35}$ The BM compartment comprises a low-oxygenic niche to ensure protection from ROS-induced stress and damage and this protection is lost during the ageing process. ${ }^{36}$ ROS is also known to play a critical role in lineage decision of myeloid progenitors and can promote differentiation into GMP. ${ }^{37}$ Therefore, it is possible that the increased ROS in Casp2 ${ }^{-1-}$ mice contributes to the observed agerelated DNA damage and aneuploidy, as well as the enhanced myeloid skewing with age.

As the precise mechanisms underlying haematopoietic ageing remain poorly understood, the Casp $2^{-1}$ mice may provide a useful tool to further develop this hypothesis. Additional studies will be important to determine the molecular pathway by which caspase-2 regulates HSC and progenitor genomic stability and differentiation, and to assess any increase in susceptibility of aged Casp $2^{-/}$mice to leukaemia. Such knowledge will provide insight into the causative factors associated with increased incidence of haematological malignancies with ageing.

\section{Materials and Methods}

Mice. Caspase-2 knockout (Casp2 $\left.{ }^{-/}\right)$mice have been described previously. ${ }^{18,25}$ These mice have been backcrossed to a C57BL/6 background for at least 20 generations. Animals were maintained in specific pathogen-free conditions in a $12 \mathrm{~h} / 12 \mathrm{~h}$ light/dark cycle and all animal breeding and studies were approved by the SA Pathology/Central Northern Adelaide Health Services Animal Ethics Committee.

Flow cytometry analysis. BM cells were stained and analysed according to previously described protocols. ${ }^{26,27}$ For the stem cell and lymphoid progenitor panel, $8 \times 10^{5}$ of BM cells were stained with the following antibodies: FITC antimouse CD48 (Biolegend, San Diego, CA, USA), Anti-mouse CD135 (Flt3) (eBioscience, San Diego, CA, USA), APC anti-mouse CD150 (SLAM) (BioLegend), Anti-mouse CD117 (c-Kit) APC-eFluor (eBioscience), Pacific Blue anti-mouse Ly6A/E (Sca-1) (BioLegend), Biotin anti-mouse CD127 (IL-7R $\alpha$ ) (BioLegend), StrepAvidin-PECy7 (BioLegend) and lineage antibodies (CD3e-PECy5 (eBioscience), B220-PECy5 (BioLegend), Gr1-PECy5 (BioLegend), Mac1-PECy5 
(BioLegend) and TER-119-PECy5 (BioLegend)). The gating strategy for stem and lymphoid progenitor cell analysis is shown in Supplementary Figure S2.

For the myeloid progenitor panel, $8 \times 10^{5} \mathrm{BM}$ cells were stained with the following antibodies: FITC rat anti-mouse CD41 (BD Pharmingen, San Jose, CA, USA), PE rat anti-mouse CD16/32 (BD Pharmingen), APC anti-mouse CD150 (SLAM) (BioLegend), Anti-mouse CD117 (c-Kit) APC-eFluor (eBioscience), Pacific Blue anti-mouse Ly-6A/E (Sca-1) (BioLegend), PE/Cy7 anti-mouse CD105 (BioLegend) and lineage antibodies (PE/Cy5 anti-mouse CD3c (eBioscience), PE/Cy5 anti-mouse CD11b (BioLegend), PE/Cy5 anti-mouse CD45R/B220 (BioLegend), PE/Cy5 antimouse Ly-6G/Ly-6C (Gr-1) (BioLegend) and PE/Cy5 anti-mouse TER-119 (BioLegend)). Samples were run on Fortressa Flow Cytometer (BD Biosciences, San Jose, CA, USA) and data analysis was performed using FCS Express Flow Cytometry Research Edition Version 4 (DeNovo Software, Glendale, CA, USA). The gating strategy for myeloid progenitor cell analysis is shown in Supplementary Figure S3.

CFU assay. Methylcellulose colony-forming assays were performed using MethoCult GF M3434 (Stem Cell Technologies, Vancouver, BC, Canada) according to the manufacturer's protocol. ${ }^{38}$ Briefly, $1 \times 10^{4} \mathrm{BM}$ cells were seeded in triplicate. CFU-GM and spontaneous BFU-E colonies, $>50$ cells in size, were counted at day 7 post seeding. Representative photographs for colony morphology were taken using an Olympus CK2 microscope (Olympus Corporation, Japan, Tokyo) and DP11 camera system at a magnification of $100 x$. For serial replating assays, cells from the triplicate day 7 post seeding were harvested and washed 3 times in IMDM media to wash away the MethoCult. Again, $1 \times 10^{4} \mathrm{BM}$ cells were seeded in triplicate. At day 7 post seeding, colonies were scored and the same procedures were repeated.

LTC-IC assay. LTC-IC assay was carried out as described previously. ${ }^{39}$ BM cells were plated in duplicate on AFT024 feeder cell layers (irradiated with $20 \mathrm{~Gy}$ ) established in 24-well plates in MyeloCult M5300 (Stem Cell Technologies) freshly supplemented with $10^{-6} \mathrm{M}$ hydrocortisone. Cultures were maintained for 5 weeks at $37^{\circ} \mathrm{C}$ with weekly half-media changes before seeding the harvested cells for CFU assays. Representative colony images were taken using a stereomicroscope (Nikon, Tokyo, Japan).

Cytogenetic and DNA damage analysis. Preparation of chromosome spreads and DNA damage analysis was carried out as previously described. ${ }^{11,15}$ Chromosome spreads were prepared by adding colcemid $(20 \mathrm{ng} / \mathrm{ml}) 4 \mathrm{~h}$ before harvesting cells by trypsinization, hypotonic treatment $(0.075 \mathrm{M} \mathrm{KCl})$ and fixation in fresh ice-cold Carnoy's fixative (methanol/glacial acetic acid at $3: 1$ ) for $10 \mathrm{~min}$ at $37^{\circ} \mathrm{C}$. Cells were centrifuged at 1000 r.p.m., washed three times in Carnoy's fixative and dropped onto wet glass slides, air dried and then placed in a $60^{\circ} \mathrm{C}$ oven overnight. Cells were stained with DAPI and chromosomes quantitated by epifluorescence microscopy (model BX51; Olympus) and camera (UCMAD3/ CVM300, Olympus). Cells were visualized under $40 x$ or $100 \times$ ULAPO objective lens with $N A=1.5$. Images were processed using Olysia BioReport Software (Olympus) and manually merged using Adobe Photoshop 6.0 software (Adobe Systems Inc., San Jose, CA, USA).

For DNA damage analysis, primary BM cells $\left(1 \times 10^{5}\right)$ were fixed in $4 \% \mathrm{w} / \mathrm{v}$ paraformaldehyde for 15 min at $4{ }^{\circ} \mathrm{C}$, permeabilized with $0.25 \% \mathrm{v} / \mathrm{v}$ Triton X-100/PBS for $10 \mathrm{~min}$ at RT and then blocked in $1 \% \mathrm{w} / \mathrm{v}$ BSA/PBS for $1 \mathrm{~h}$ at RT. Cells were incubated with anti-phospho-histone H2AX (Ser139) primary antibody (Cell Signaling Technology, Danvers, MA, USA) diluted in blocking buffer, overnight at $4{ }^{\circ} \mathrm{C}$. Following 3 washes in blocking buffer, cells were incubated with donkey anti-rabbit Alexa Fluor 488 secondary antibody (Life Technologies, Carlsbad, CA, USA) diluted 1:1000 in blocking buffer for $1 \mathrm{~h}$ at RT. Following antibody incubations and washes in PBS, cells were counterstained with $2 \mu \mathrm{g} / \mathrm{ml}$ DAPI for 5 min and mounted with ProLong Gold Antifade Reagent (Thermo Fisher Scientific, Waltham, MA, USA). At least 100 cells were scored for each sample. Fluorescent images were captured using a LSM 800 confocal laser scanning microscope (Zeiss, Oberkochen, Germany).

Giemsa staining. BM cell cytospins were stained using Aerospray Hematology Pro (EliTech Group, Puteaux, France) by the Department of Clinical Pathology, SA Pathology (Adelaide, SA, Australia) and analysed by an expert haematologist. The images were captured using an epifluorescence microscopy (model BX51; Olympus) and camera (UCMAD3/CVM300, Olympus).
Statistical analysis of data. Statistical analysis was carried out in GraphPad Prism, Version 6.05 (GraphPad, GraphPad Inc., La Jolla, CA, USA). Student's t-test was used for statistical significance analysis to determine $P$-values between each genotype, unless otherwise stated. Data are expressed as mean \pm S.E.M. $P<0.05$ was considered significant.

\section{Conflict of Interest}

The authors declare no conflict of interest.

Acknowledgements. We thank staff at the SA Pathology animal resource facility for help in maintaining the mouse strains and members of our laboratory for discussions and useful comments. Funding for this work was provided by the National Health and Medical Research Council project grant (1043057) and a Senior Principal Research Fellowship (1103006) to SK. SD was supported by a UniSA Presidential Award.

\section{Author contributions}

SD, LD, DKH and SK conceptualized the project; SD performed experiments; NS provided technical help; SD, DKH and SK analysed data and wrote the paper; NHS and RJDA analysed flow data and reviewed the manuscript; LD, DKH and SK supervised the study.

1. Kumar S, Kinoshita M, Noda M, Copeland NG, Jenkins NA. Induction of apoptosis by the mouse Nedd2 gene, which encodes a protein similar to the product of the Caenorhabditis elegans cell death gene ced-3 and the mammalian IL-1 beta-converting enzyme. Genes Dev 1994; 8: 1613-1626.

2. Shalini S, Dorstyn L, Dawar S, Kumar S. Old, new and emerging functions of caspases. Cell Death Differ 2015; 22: 526-539.

3. Kumar S. Caspase 2 in apoptosis, the DNA damage response and tumour suppression: enigma no more? Nat Rev Cancer 2009; 9: 897-903.

4. Puccini J, Dorstyn L, Kumar S. Caspase-2 as a tumour supressor. Cell Death Differ 2013; 20: 1133-1139.

5. Kumar S, White DL, Takai S, Turczynowicz S, Juttner CA, Hughes TP. Apoptosis regulatory gene NEDD2 maps to human chromosome segment $7 q 34-35$, a region frequently affected in haematological neoplasms. Hum Genet 1995; 95: 641-644.

6. Hofmann WK, de Vos S, Tsukasaki K, Wachsman W, Pinkus GS, Said JW et al. Altered apoptosis pathways in mantle cell lymphoma detected by oligonucleotide microarray. Blood 2001; 98: 787-794.

7. Holleman A, den Boer ML, Kazemier KM, Beverloo HB, von bergh AR, Janka-Schaub GE et al. Decreased PARP and procaspase-2 protein levels are associated with cellular drug resistance in childhood acute lymphoblastic leukemia. Blood 2005; 106: 1817-1823.

8. Johansson B, Mertens F, Mitelman F. Cytogenetic deletion maps of hematologic neoplasms: circumstantial evidence for tumor suppressor loci. Genes Chromosomes Cancer 1993; 8: 205-218.

9. Ho LH, Taylor R, Dorstyn L, Cakouros D, Bouillet P, Kumar S. A tumor suppressor function for caspase-2. Proc Natl Acad Sci USA 2009; 106: 5336-5341.

10. Manzl C, Peintner L, Krumschnabel G, Bock F, Labi V, Drach M et al. PIDDosomeindependent tumor suppression by Caspase-2. Cell Death Differ 2015; 19: 1722-1732.

11. Puccini J, Shalini S, Voss AK, Gatei M, Wilson CH, Hiwase DK et al. Loss of caspase-2 augments lymphomagenesis and enhances genomic instability in Atm-deficient mice. Proc Natl Acad Sci USA 2013; 110: 19920-19925.

12. Parsons MJ, McCormick L, Janke L, Howard A, Bouchier-Hayes L, Green DR. Genetic deletion of caspase-2 accelerates MMTV/c-neu-driven mammary carcinogenesis in mice. Cell Death Differ 2013; 20: 1174-1182.

13. Terry MR, Arya R, Mukhopadhyay A, Berrett KC, Clair PM, Witt B et al. Caspase-2 impacts lung tumorigenesis and chemotherapy response in vivo. Cell Death Differ 2015; 22: 719-730.

14. Shalini S, Nikolic A, Wilson $\mathrm{CH}$, Puccini J, Sladojevic N, Finnie J et al. Caspase-2 deficiency accelerates chemically induced liver cancer in mice. Cell Death Differ 2016; 23: 1727-1736.

15. Dorstyn L, Puccini J, Wilson CH, Shalini S, Nicola M, Moore S et al. Caspase-2 deficiency promotes aberrant DNA-damage response and genetic instability. Cell Death Differ2012; 19: 1288-1298.

16. Dorstyn L, Puccini J, Nikolic A, Shalini S, Wilson CH, Norris MD et al. An unexpected role for caspase-2 in neuroblastoma. Cell Death Dis 2014; 5 : e1383.

17. Peintner L, Dorstyn L, Kumar S, Aneichyk T, Villunger A, Manzl C. The tumor-modulatory effects of Caspase-2 and Pidd1 do not require the scaffold protein Raidd. Cell Death Differ 2015; 22: 1803-1811.

18. Shalini S, Dorstyn L, Wilson C, Puccini J, Ho L, Kumar S. Impaired antioxidant defence and accumulation of oxidative stress in caspase-2-deficient mice. Cell Death Differ 2012; 19: $1370-1380$. 
19. Zhang $Y$, Padalecki SS, Chaudhuri AR, De Waal E, Goins BA, Grubbs B et al. Caspase-2 deficiency enhances aging-related traits in mice. Mech Ageing Dev 2007; 128 : 213-221.

20. Shalini S, Puccini J, Wilson CH, Finnie J, Dorstyn L, Kumar S. Caspase-2 protects against oxidative stress in vivo. Oncogene 2015; 34: 4995-5002.

21. Wilson $\mathrm{CH}$, Shalini S, Filipovska A, Richman TR, Davies S, Martin SD et al. Age-related proteostasis and metabolic alterations in Caspase-2-deficient mice. Cell Death Dis 2015; 6 : e1597.

22. Wilson $\mathrm{CH}$, Nikolic A, Kentish SJ, Shalini S, Hatzinikolas G, Page AJ et al. Sex-specific alterations in glucose homeostasis and metabolic parameters during ageing of caspase-2deficient mice. Cell Death Discov 2016; 2: 16009.

23. Morrison SJ, Wandycz AM, Akashi K, Globerson A, Weissman IL. The ageing of hematolopoietic stem cells. Nat Med 1996; 2: 1011-1016.

24. Geiger H, de Haan G, Florian MC. The ageing haematopoietic stem cell compartment. Nat Rev Immunol 2013; 13: 376-389.

25. O'Reilly LA, Ekert P, Harvey N, Marsden V, Cullen L, Vaux DL et al. Caspase-2 is not required for thymocyte or neuronal apoptosis even though cleavage of caspase-2 is dependent on both Apaf-1 and caspase-9. Cell Death Differ 2002; 9: 832-841.

26. Pronk CJ, Bryder D. Flow cytometry-based identification of immature myeloerythroid development. Meth Mol Biol 2011; 699: 275-293.

27. Akashi K, Traver D, Miyamoto T, Weissman IL. A clonogenic common myeloid progenitor that gives rise to all myeloid lineages. Nature 2000; 404: 193-197.

28. Genovese G, Ak Kahler, Handsaker RE, Lindberg J, Rose SA, Bakhoum SF et al. Clonal hematopoiesis and blood-cancer risk inferred from blood DNA sequence. NEng J Med 2014; 371: 2477-2487.

29. Rossi DJ, Jamieson $\mathrm{CH}$, Weissman IL. Stems cells and the pathways to aging and cancer Cell 2008; 132: 681-696.

30. Rossi DJ, Bryder D, Zahn JM, Ahlenius H, Sonu R, Wagers AJ et al. Cell intrinsic alterations underlie hematopoietic stem cell aging. Proc Natl Acad Sci USA 2005; 102 9194-9199.

31. Xie M, Lu C, Wang J, McLellan MD, Johnson KJ, Wendl MC et al. Age-related mutations associated with clonal hematopoietic expansion and malignancies. Nat Med 2014; 20 1472-1478.

32. Bakhoum SF, Danilova OV, Kaur P, Levy NB, Compton DA. Chromosomal instability substantiates poor prognosis with diffuse large B-cell lymphoma. Clin Cancer Res 2011; 17: 7704-7711.
33. Birkbak NJ, Eklund AC, Li Q, McClelland SE, Endesfelder D, Tan P et al. Paradoxical relationship between chromosomal instabiliy and survival outcome in cancer. Cancer Res 2011; 71: 3447-3452.

34. Dawar S, Lim Y, Puccini J, White M, Thomas $P$, Bouchier-Hayes $L$ et al. Caspase-2-mediated cell death is required for deleting aneuploid cells. Oncogene 2016; doi:10.1038/onc.2016.423

35. Li T, Zhou ZW, Ju Z, Wang ZQ. DNA damage response in hematopoietic stem cell ageing. Genom Proteom Bioinform 2016; 14: 147-154.

36. Porto ML, Rodriguez BP, Menezes TN, Ceschim SL, Casarini DE, Gava AL et al. Reactive oxygen species contribute to dysfunction of bone marrow hematopoietic stem cells in aged C57BL/6 J mice. J Biomed Sci 2015; 22: 97.

37. Shinohara A, Imai Y, Nakagawa M, Takahashi T, Ichikawa M, Kurokawa M. Intracellular reactive oxygen species mark and influence the megakaryocytic-erythrocyte progenitor fate of common myeloid progenitor. Stem Cells 2014; 32: 548-557.

38. Metcalf D, Nicola NA. Direct proliferative actions of stem cell factor on murine bone marrow cells in vitro: effects of combination with colony-stimulating factors. Proc Natl Acad Sci USA. 1991; 88: 6239-6243.

39. Nievergall E, Ramshaw HS, Yong ASM, Biondo M, Busfield SJ, Vairo G et al. Monoclonal antibody targeting of IL-3 receptor $\alpha$ with with CSL362 effectively depletes CML progenitor and stem cells. Blood 2014; 123: 1218-1228.

(i) Cell Death and Disease is an open-access journal published by Nature Publishing Group. This work is licensed under a Creative Commons Attribution 4.0 International License. The images or other third party material in this article are included in the article's Creative Commons license, unless indicated otherwise in the credit line; if the material is not included under the Creative Commons license, users will need to obtain permission from the license holder to reproduce the material. To view a copy of this license, visit http://creativecommons.org/licenses/by/4.0/

(C) The Author(s) 2016

Supplementary Information accompanies this paper on Cell Death and Disease website (http://www.nature.com/cddis) 